\title{
ЭКОНОМИЧЕСКИЙ ПОТЕНЦИАЛ ПРЕДПРИЯТИЯ: ПОДХОДЫ К ОЦЕНКЕ
}

\section{ECONOMIC POTENTIAL OF THE COMPANY: EVALUATION APPROACHES}

\section{N. Skvortsova}

L. Filimonova

Summary. This publication discloses the features of using statistical tools as a way to conduct a comprehensive assessment of the economic potential through the prism of an array of parameters as applied to a business entity at the micro level. The proposed approach to assessing the economic potential of an enterprise reveals the strengths and weaknesses of the subject of assessment through strata (blocks, subpotentials) and synthesizes the traditional statistical apparatus, the method of expert estimates and the cumulative compromise scheme in the form of additive convolution, as well as resource approaches (potentials) to assessment and management economic potential. The results of the study can be used to increase the predictability of doing business in conditions of risk and uncertainty of the macroenvironment and make the process of managing potential systemic.

Keywords: economic potential, multi-level filtering, stratified sampling, subpotentials, statistical tools, enterprise.

\author{
Скворцова Надежда Константиновна \\ Д.э.н., профессор, ФГБОУ ВО «Тюменский \\ индустриальный университет» \\ skvortsovank@tyuiu.ru \\ Филимонова Лариса Акрамовна \\ К.э.н., дочент, ФГБОУВО «Тюменский \\ индустриальный университет» \\ Filimonovala@tyuiu.ru
}

Аннотация. В настоящей публикации раскрыты особенности применения статистического инструментария, как способа проведения комплексной оценки экономического потенциала предприятия через призму массива параметров применительно к хозяйствующему субъекту на микроуровне. Предлагаемый подход к оценке экономического потенциала предприятия раскрывает сильные и слабые позиции субъекта оценки через страты (блоки, субпотенциалы) и синтезирует традиционный статистический аппарат, метод экспертных оценок и кумулятивную схему компромисса в виде аддитивной свертки, а также ресурсные подходы (потенциалы) к оценке экономического потенциала. Результаты исследования могут быть использованы для повышения предсказуемости ведения бизнеса в условиях риска и неопределенности макроокружения и придания процессу оценки потенциала системности.

Ключевые слова: экономический потенциал, многоуровневая фильтрация, стратифицированная выборка, субпотенциалы, статистический инструментарий, предприятие.

остановиться на теоретических подходах к определению категории экономического потенциала в исследованиях отечественных и зарубежных ученых-экономистов. На сегодняшний день существует множество различных определений и трактовок понятий «потенциал», «ресурсы», «экономический потенциал», «ресурсный потенциал», остановимся лишь на наиболее привлекательных трактовках (табл. 1). Необходимо остановиться на уточнении понятия потенциала.

Принципиальным отличием между терминами «ресурсы» и «потенциал» является то, что ресурсы существуют независимо от субъектов экономической деятельности, а потенциал отдельного предприятия, общества в целом неотделим от субъектов деятельности. Т. е. «потенциал», кроме материальных и нематериальных средств, включает способности работника, коллектива, предприятия, общества в целом к эффективному использованию имеющихся средств или ресурсов. Термин «ресурсный потенциал» в научных исследованиях используется в основном в отношении субъектов Российской Федерации, крупных экономических районов, страны в целом. Но, поскольку одними из главных составляющих 
Таблица 1. Сводка понятийного аппарата

\begin{tabular}{|c|c|}
\hline Автор (источник) & Содержание понятия \\
\hline $\begin{array}{l}\text { Большой экономический } \\
\text { словарь }\end{array}$ & $\begin{array}{l}\text { «Потенциал», без определения его видов, представляет собой совокупность средств, запасов, } \\
\text { источников, имеющихся в наличии, которые могут быть мобилизованы, приведены в действие, } \\
\text { использованы для достижения цели. Иногда термин потенциал трактуется как «возможности» или } \\
\text { «способность», но в каждый определенный момент времени можно обозначить совокупность } \\
\text { средств, определяющих данную способность [2]. }\end{array}$ \\
\hline $\begin{array}{l}\text { Словарь русского языка } \\
\text { С.И. Ожегова, российский } \\
\text { энциклопедический словарь } \\
\text { А.М. Прохорова }\end{array}$ & $\begin{array}{l}\text { Потенциал - «величина, характеризующая широкий класс силовых полей в данной точке, } \\
\text { открывающая целый ряд понятий в физике, химии, математике» }[3,4] .\end{array}$ \\
\hline К.М. Миско & $\begin{array}{l}\text { Потенциал — «предел человеческих познаний о внутренних, скрытых возможностях результативного } \\
\text { использования изучаемого объекта, которые могут быть количественно оценены и в конечном счете } \\
\text { реализованы при идеальных условиях практической деятельности» [5]. }\end{array}$ \\
\hline А.Н. Азрилиян & Совокупность имеющихся средств, возможностей в какой-либо области [2]. \\
\hline А.Н. Люкшинова & $\begin{array}{l}\text { Потенциал предприятия представляет собой совокупность его возможностей по выпуску продукции } \\
\text { (оказанию услуг) [6]. }\end{array}$ \\
\hline Т.Ф. Рябова & $\begin{array}{l}\text { «Потенциал - совокупность имеющихся факторов производства, интеллекта, производственных } \\
\text { резервов и возможностей, способных обеспечить выпуск высококачественных товаров, необходимых } \\
\text { для удовлетворения всесторонних запросов различных категорий населения страны» [7] }\end{array}$ \\
\hline Т.Г. Храмцова & $\begin{array}{l}\text { «Птенциал - это не только и не просто количество ресурсов, но и заключенная в них возможность } \\
\text { развития системы в заданном направлении [8]. Возможности должны быть реализованы. Как } \\
\text { в механике потенциальная энергия реализуется в кинетическую, так и в экономике реализация } \\
\text { потенциала находит воплощение в результатах деятельности». }\end{array}$ \\
\hline Ю.Н. Клепиков & $\begin{array}{l}\text { «...величина потенциала предприятия определяется количеством ресурсов, которыми располагает } \\
\text { предприятие, и условиями, позволяющими достичь наиболее полного и рационального их } \\
\text { использования» [9]. }\end{array}$ \\
\hline
\end{tabular}

ресурсного потенциала этих субъектов являются предприятия, которые обеспечивают производство товаров и услуг, представляется вполне обоснованным применить этот термин в отношении предприятия. Следует отметить, что в теоретическом и практическом аспектах категория ресурсный потенциал на уровне предприятия изучена недостаточно, хотя в научной литературе на макроуровне обсуждается довольно широко. Для определения ресурсного потенциала предприятия как объекта исследования экономической науки необходимо остановиться на теоретических подходах к этому термину в исследованиях ученых-экономистов. Так, В.А. Свободин характеризует ресурсный потенциал как «совокупность имеющихся в распоряжении предприятия ресурсов (земельные, трудовые, материальные)» [10].

По мнению Л.Г.Окороковой ресурсный потенциал предприятия представляет собой совокупность всех ресурсов предприятия, обеспечивающих возможность получения максимального экономического эффекта в заданный момент времени [11].

Обобщение теоретических взглядов ученых позволяет констатировать тот факт, что в экономической литературе существуют различные позиции к содержанию категории «ресурсный потенциал». При этом одни считают, что эта категория представляет собой конгломерат ресурсов, без учета их качественной стороны. Другие полагают, что ресурсный потенциал представляет собой материальную основу производства, но в статике, т.е. до момента их вовлечения в производственный процесс. Третьи - не учитывают целевое назначение ресурсного потенциала.

В результате проведенного изучения и систематизации различных научных взглядов, приходим к выводу, что под ресурсным потенциалом предприятия следует понимать совокупность имеющихся видов ресурсов, сопряженных между собой, использование которых позволяет достичь экономического эффекта, что в целом представляет собой экономический потенциал предприятия. Причем, одни считают, в частности А.Г. Фонотов, что категория «ресурсный потенциал» шире экономического потенциала, при этом отличие заключается в том, что последний не учитывает долгосрочную перспективу [12]. На наш взгляд, понятие «экономический потенциал» шире ресурсного потенциала, а последний является его составной частью, кроме того, ресурсный потенциал не включает в свою структуру производственно-технологические, организационные и экономические связи.

При этом внутренняя среда никак не коррелирует с внешними индикаторами и параметрами рынка. Изло- 
женное выше подчеркивает актуальность вопроса недостатка универсального метода оценки субпотенциалов внутренней и внешней среды предприятия, результаты оценки которых, в свою очередь, выступали бы индикаторами для принятия своевременных управленческих решений в условиях неопределенности внешней среды субъекта. Проблема объясняется сложностью применения математических моделей, позволяющих «перевести на язык цифр и формул экономические явления и получить обоснованные расчетами результаты» [13, с. 139], и выбора наиболее значимого подхода и модели расчета некоего коэффициента, претендующего на звание комплексного.

Результаты предлагаемого комплексного подхода к оценке экономического потенциала предприятия через алгоритм стратифицированной выборки традиционных показателей классического анализа финансово-хозяйственной деятельности составят базис информационного ресурса для корректировки стратегии, программы и планов дальнейшего развития субъекта.

Обобщение вышеизложенного позволяет сделать вывод о том, что в период перехода от стратегии развития к стратегии выживания как никогда актуальны вопросы изучения экономического потенциала предприятия в целях принятия адекватных управленческих решений по сохранению бизнеса и рабочих мест в регионе. В связи с чем, авторы настоящей публикации, под экономическим потенциалом понимают совокупность имеющихся ресурсов, разгруппированных по стратам (блокам), сопряженных между собой, использование которых позволяет достичь экономического эффекта в условиях агрессивной внешней среды и ограниченности доступа к внешним финансовым ресурсам.

Для того чтобы оценить экономический потенциал предприятия, необходимо иметь четкие признаки его идентификации и классификацию влияющих на него факторов внешней среды. Важную роль при исследовании страт (блоков) экономического потенциала играет системный подход, при котором последний изучается как целостная система, состоящая из подсистем, представленных стратами (блокам) различного порядка, образующих уровни системы. Большинство авторов сходится в едином мнении о необходимости комплексной оценки всех потенциалов субъекта посредством разработки некоего сводного (синтетического, интегрального, комплексного и пр.) показателя оценки, включающего в себя все стороны производственно-хозяйственной деятельности предприятия, его имущественного и финансового обеспечения.

По мнению большинства ученых, динамика сводного показателя экономического потенциала предприятия позволяет заблаговременно сигнализировать о возможных потерях, либо приобретениях, что в свою очередь повышает результативность принимаемых управленческих решений, направленных на сохранение и преумножение имеющихся потенциалов.

Теоретико-методической основой настоящего исследования послужили фундаментальные и прикладные научные труды отечественных и зарубежных авторов в области экономической теории, теории инвестиций, статистики и экономики. Для обоснования результатов исследования использовались методы экономико-математического моделирования, элементы ресурсного и системного подходов, статистические методы. Подводя итоги проведенного анализа теоретического и практического материала по имеющимся печатным и электронным ресурсам, авторы статьи сходятся во мнении о целесообразности проведения оценки не по комплексу показателей экономического потенциала, а по выделенным его стратам (блокам), которым присваиваем наименование «субпотенциал».

Разработка универсальной модели соответствующего субпотенциала предприятия с последующим их синтезом позволит повысить уровень индикативности и своевременности принятия предупреждающих управленческих, антикризисных и прочих мер по сохранению бизнеса в современных условиях неопределенности на российском рынке товаров, работ, услуг отечественного производителя. Обобщив сложившуюся практику оценки уровня экономического потенциала предлагаем следующую последовательность аналитических изысканий, синтезирующую статистический инструментарий регрессии в рядах динамики с кросс-секционной регрессией стационарной системы:

1. формирование массива традиционных показателей классического анализа финансово-хозяйственной деятельности предприятия и статистических показателей, отражающих социально-экономическое развитие региона за длительный временной диапазон согласно открытым источникам информации;

2. стратифицированная группировка массива показателей с выделением следующих субпотенциалов:

а) рисковая составляющая потенциала внешней среды предприятия на территории заданного региона,

б) экономическая составляющая потенциала внутренней среды предприятия: производственно-технологический; имущественный; финансовый потенциалы;

3. стратифицированная выборка статистических показателей из заданного массива, которым присваивается статус оценочного критерия с помо- 


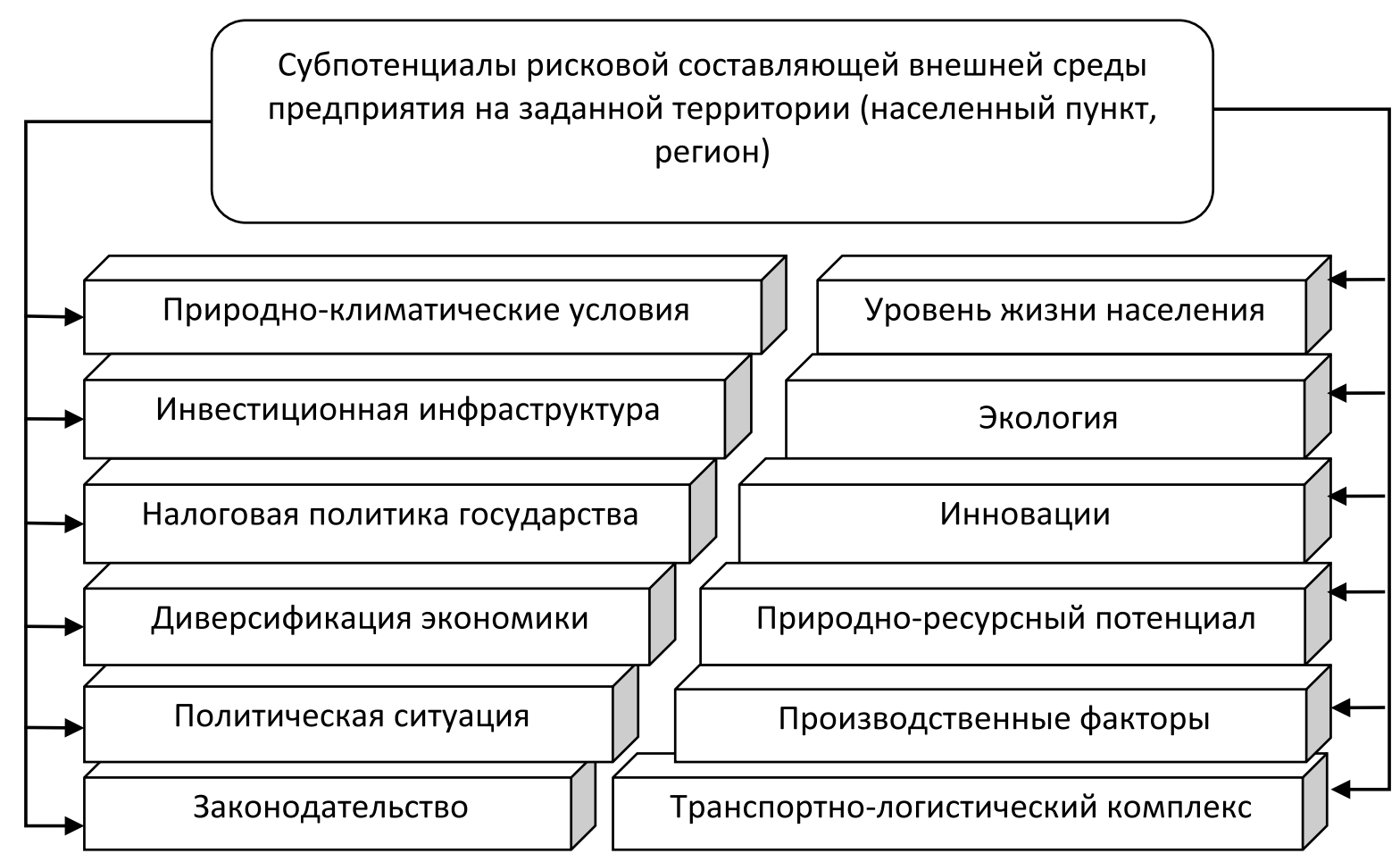

Рис. 1. Субпотенциалы рисковой составляющей потенциала внешней среды предприятия

щью статистического инструментария корреляционного анализа;

4. формирование по результатам выборки оценочных критериев параметрических моделей оценки экономической и рисковой составляющих (форм.1-5);

5. апробация параметрических моделей на уровне предприятия применительно к особенностям социально-экономического развития заданного региона;

6. интерпретация результатов апробации параметрических моделей оценки экономического потенциала предприятия.

Остановимся на отдельных позициях предлагаемых этапов оценки потенциала предприятия. На рис. 1 представляем свод ключевых субпотенциалов рисковой составляющей потенциала внешней среды.

Экологический субпотенциал рисковой составляющей потенциала внешней среды предприятия предлагается выделить в самостоятельный блок, а не в разрезе производственно-технологического блока, поскольку современному производственному предприятию необходимо уделять должное внимание мероприятиям, направленным на снижение негативного воздействия производственных факторов на окружающую среду, с одной стороны, и учитывать влияние загрязнения окружающей среды на производственный потенциал ускорением его износа и преждевременным выходом из стоя основных производственных фондов, с другой.

Финансовый блок субпотенциала экономической составляющей потенциала внутренней среды предлагаем формировать с позиции расчета показателей ликвидности, платежеспособности, финансовой устойчивости баланса, доходности, оборачиваемости активов предприятия, в качестве примера рекомендуем публикации следующих авторов [13, 14].

Прежде чем приступать к разработке параметрических моделей экономической и рисковой составляющих экономического потенциала предприятия введем следующие условные обозначения: $t=\overline{1, T}$ - период времени (наблюдения); $l=\overline{1, L}-$ направления размещения инвестиционного капитала (технологическая либо воспроизводственная структура капитальных вложений проектируемого либо действующего предприятия); $j=\overline{1, m}$ - субпотенциалы экономической составляющей бухгалтерской (финансовой) отчетности; $F_{t q L}-$ интегральный критерий оценки экономического потенциала предприятия в t-й период при q-ом объеме выпуска продукции и заданному значению цены (Ц); $\beta_{t}$ - экономическая составляющая внутренней среды предприятия в t-й период наблюдения; Risk взвешенная составляющая внешней среды предприятия

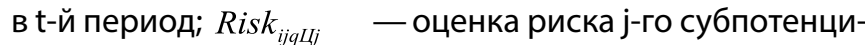
ала для t-ого периода наблюдения; $\lambda_{j}$ - значимость ј-го 


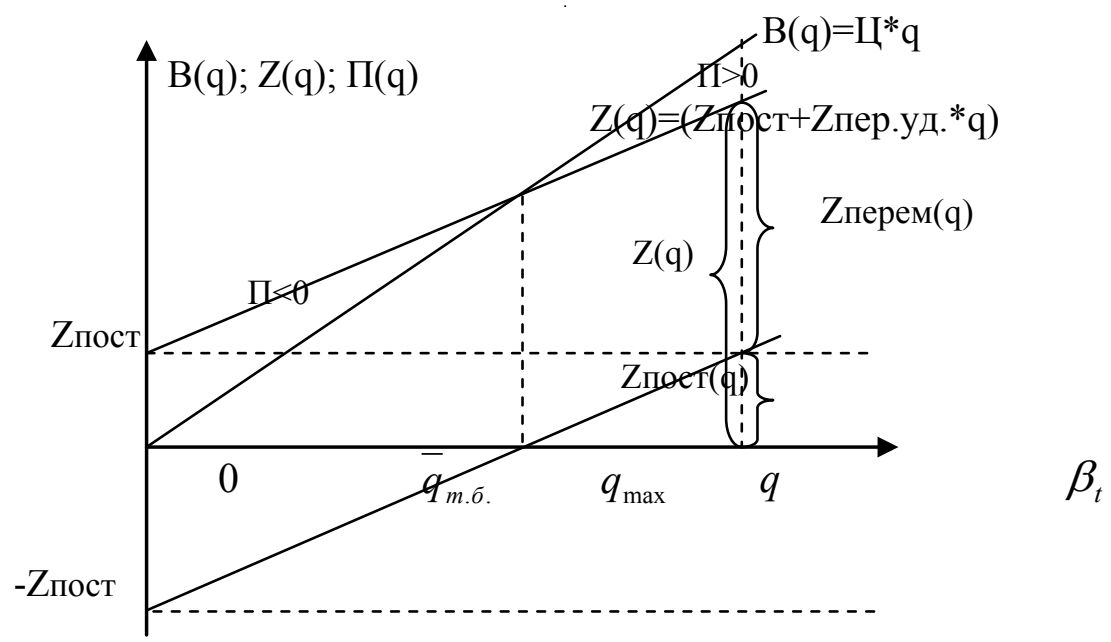

Рис. 2. Схема поиска критического значения натурального объема производства для расчета экономической составляющей потенциала внутренней среды предприятия

субпотенциала внутренней среды предприятия в оценке риска воздействия внешней среды, задаваемая эвристическим способом (экспертно); $V A P_{t}=B(q)-M P-$ добавленная стоимость продукта (value added product) в t-й период; $h_{l t}$ - объем инвестиций в $l$-ую сферу в t-й период; $X_{i j t}$ - значение і-го показателя ј-ого субпотенциала внутренней среды в t-ом периоде времени при q-том объеме выпуска продукции; $B(q)=Z(q)+\Pi(q)$ - выручка от реализации предполагаемого объема выпуска продукции; $Z(q)=(Z n o c m+Z n e p . y d$. *q) - затраты предприятия при соответствующем объеме выпуска продукции (q); $Z$ переем - затраты переменные, обусловленные внешними факторами, например закупочными ценами на сырье, материалы, ресурсы; средним уровнем доходов в регионе, что определяет уровень оплаты труда работников предприятия; нормативами обязательных отчислений страховых взносов в социальные фонды от начисленного уровня оплаты труда работников и пр. затратами, зависящими от количества выпущенной продукции); $M P$ - величина материальных расходов, формируемая согласно закупочных цен на сырье, материалы, ресурсы, которые устанавливают поставщики этих ресурсов; П $(q)$ - прибыль от реализации объема производимой продукции; $k(q)$ - уровень финансового коэффициента соответствующего $j$-го субпотенциала внутренней среды при соответствующем объеме выпуска продукции $(q)$.

На рис. 2 представлена стационарная схема для расчета критического объема выпуска продукции предприятия, который является базисом экономической составляющей внутренней среды экономического потенциала в на t-ом периоде времени.

Под уровнем критического объема выпуска продукции подразумеваем величину натурального выпуска продукции, при котором имеет место покрытие всех постоянных затрат, которые понесет предприятие, иными словами, тот объем производства в натуральном выражении, при котором предприятие выйдет на безубыточный уровень.

На 3 этапе стратифицированную выборку показателей предлагаем осуществлять посредством статистического инструментария корреляционного метода анализа, что позволяет ориентироваться на сущность причинно-следственных связей в заданном массиве исходных показателей. В результате применения стратифицированной выборки оценочных критериев по сформированным субпотенциалам осуществляется многоуровневая фильтрация исходного массива. Фильтрацию в выборке проходят только те показатели по субпотенциалам, которые в паре демонстрируют связь в следующих интервалах: 0,35 $\leq r x y \leq 0,75$ и -0,75 $\leq r x y \leq-0,35$. Завершающим шагом в построения конечной матрицы оценочных критериев является выбор только тех показателей по каждому субпотенциалу, которые при суммировании повторений по столбцам и строкам выдали наибольше число побед - суммарных повторений в матрице линейных коэффициентов корреляции. В результате данных итераций приходим к свертке детерминированной модели.

Детерминированная модель интегрального критерия оценки потенциала предприятия на заданной территории в общем виде:

$$
F_{t}=f\left(\beta_{q t} ; \text { Risk } k_{q t}\right) \rightarrow \underset{1 \leq t \leq T}{\operatorname{extr}}
$$

Экономическую составляющую потенциала внутренней среды предприятия предлагаем представить отношением добавленной стоимости продукции к объему 


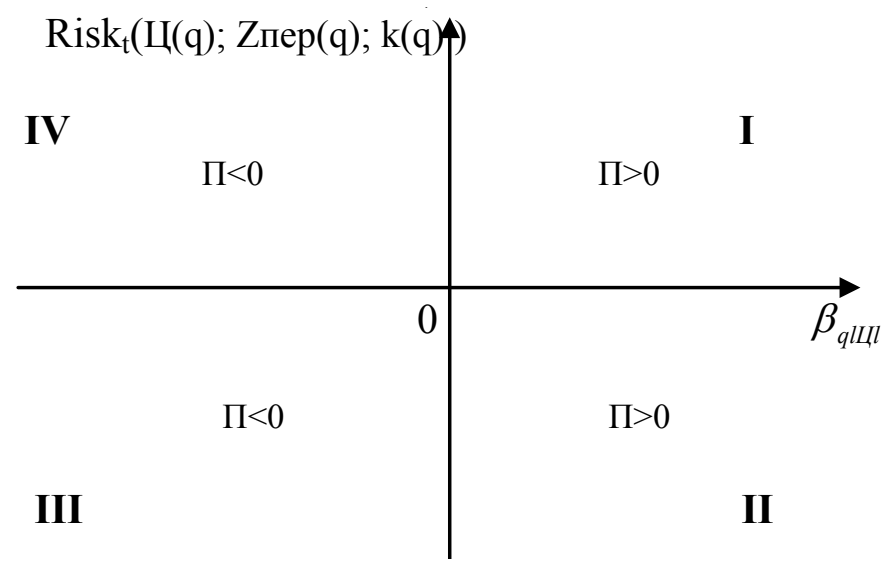

Рис. 3. Сегменты экономического потенциала предприятия

вложений в предприятие, уменьшенного на величину притока денежных средств по амортизационным отчислениям:

$$
\beta_{t}=\frac{\sum_{t=1}^{T} V A P(q)_{t}-A O_{t}}{\left|\sum_{l=1}^{L} h_{l t}-A O_{t}\right|} \rightarrow \max _{1 \leq j \leq m}
$$

Рисковую составляющую потенциала вешней среды предприятия для оценки уровня совокупного риска экономического потенциала предлагаем рассчитывать как средневзвешенную арифметическую величину по формуле:

$$
\operatorname{Risk}_{t}=\sum_{j=1}^{m} \operatorname{Risk}_{i j t} \lambda_{j} \rightarrow \min _{1 \leq t \leq T}
$$

где $R_{i j t}$ - нормализованное значение і-го показателя оценки риска ј-ого субпотенциала в t-ом периоде времени:

$$
\begin{aligned}
& R_{i j t}=\left\{\begin{array}{l}
\frac{X_{\max i}-X_{i}}{X_{\max i}-X_{\min i}}, \\
\frac{X_{i}-X_{\min t}}{X_{\max i}-X_{\min i}},
\end{array}\right. \\
& \operatorname{Risk}_{t}=\frac{\max _{1 \leq t \leq T} \frac{R_{i j t}}{\frac{1}{m} \sum_{i=1}^{n} \sum_{j=1}^{m} R_{i j t}}}{\sum_{t=1}^{T} \frac{R_{i j t}}{\frac{1}{m} \sum_{i=1}^{n} \sum_{j=1}^{m} R_{i j t}}} \rightarrow \min , i=\overline{1, n}, j=\overline{1, m} .
\end{aligned}
$$

В процессе применения статистического инструментария целесообразно первоначально те показатели $\left(X_{t}\right)$, которые выражены стоимостными единицами измерения, привести к сопоставимому уровню в динамике посредством интерполяции (дисконтирования $(\mu)$ ). В качестве коэффициента дисконтирования $(\mu)$ принимаем уровень ключевой ставки ЦБ РФ, индекс цен предприятий - производителей товаров или потребительских услуг $[14,15,16]$, что позволяет косвенно оценить скрытый уровень инфляционных явлений и ожиданий. Коэффициент соотношения рисков (см. форм. 5) определяет долю максимального отношения риска (колеблемости, волатильности, неустойчивости) субпотенциала к среднему показателю оценки риска потери экономического потенциала.

На завершающем этапе приступаем к обобщению полученных результатов апробации статистического инструментария в оценке составляющих экономического потенциала применительно к предприятию (рис. 3: по оси X откладываем значения абсциссы экономической составляющей потенциала внутренней среды потенциала (форм.2), по оси Y откладываем ординату рисковой составляющей потенциала внешней среды (форм.3)). Наиболее доступным вариантов интерпретации результатов моделирования и итераций при расчете интегральных уровней экономического потенциала предприятия является разбиение полученных результатов на группы, например посредством использования формулы Стерджесса. В результате чего, на завершающем этапе, приходим к следующим сегментам потенциала:

Возможные варианты интерпретации сегментов сведем в Табл. 2.

Результаты проведенного аналитического моделирования позволяют сформировать универсальные параметрические модели составляющих экономического потенциала предприятия, ориентированных на использование публичных сведений, размещенных в общем доступе.

Использование экономического потенциала, как одного из важнейших оценочных показателей как на ма- 
Таблица 2. Классификация уровней экономического потенциала предприятия

\begin{tabular}{|c|c|c|}
\hline $\begin{array}{l}\text { Уровень } \\
\text { потенциала }\end{array}$ & $\begin{array}{l}\text { Категория } \\
\text { (potential) }\end{array}$ & Характеристика экономического потенциала \\
\hline \multirow{2}{*}{ Высокий потенциал. } & $\begin{array}{l}\text { IP++ } \\
\text { Предприятие лидер }\end{array}$ & $\begin{array}{l}\text { Высокий уровень потенциала, регион характеризует высокую доходность } \\
\text { инвестиций в предприятие, риски завышены }\end{array}$ \\
\hline & $\begin{array}{l}\text { ІІР+Относительный уровень } \\
\text { успешности предприятия }\end{array}$ & $\begin{array}{l}\text { Выше среднего уровень экономического потенциала, регион является } \\
\text { оптимальным для инвестирования в предприятие, риски снижены }\end{array}$ \\
\hline \multirow[t]{2}{*}{ Средний потенциал. } & $\begin{array}{l}\text { ॥Р } \\
\text { Относительный уровень } \\
\text { успешности предприятия }\end{array}$ & $\begin{array}{l}\text { Средний уровень потенциала, обеспечивается средний уровень доходности, } \\
\text { риски оптимальные }\end{array}$ \\
\hline & $\begin{array}{l}\text { IIP- } \\
\text { Предприятие перспективно }\end{array}$ & $\begin{array}{l}\text { Потенциал ниже среднего, вложения в предприятие в данном регионе } \\
\text { рискованны }\end{array}$ \\
\hline \multirow{2}{*}{ Низкий потенциал } & $\begin{array}{l}\text { IIІР- } \\
\text { Предприятие перспективно } \\
\text { в другом регионе }\end{array}$ & $\begin{array}{l}\text { Низкий потенциал, вложения в предприятие в данном регионе } \\
\text { нежелательны, предприятие характеризуется убыточностью }\end{array}$ \\
\hline & $\begin{array}{l}\text { IVP- } \\
\text { Предприятие не перспективно }\end{array}$ & $\begin{array}{l}\text { Низкий потенциал, вложения в предприятие не допустимы, высокий уровень } \\
\text { рискованности, вложения в зоне не возврата }\end{array}$ \\
\hline
\end{tabular}

кро- (регион, государство), так и на микроуровне (предприятие), становится все более обоснованным в силу того, что его сущность и назначение обеспечивают базис для построения объективных прогнозов развития субъекта исследования и возможность проведения параметрического анализа тенденций и перспектив его развития, своевременно принимать корректирующие мероприятия в части управления либо предотвращения неблагоприятных изменений в заданном массиве параметров. В настоящей публикации раскрыты особенности применения статистического инструментария как способа проведения комплексной оценки экономического потенциала через призму массива параметров применительно к хозяйствующему субъекту на микроуровне - предприятия. Комплексность оценки раскрыта через алгоритм стратифицированной выборки, которая позволяет весь перечень традиционных показателей классического анализа финансово-хозяйственной деятельности свести в страты (блоки, группы). Данный подход к оценке экономического потенциала предприятия раскрывает сильные и слабые позиции субъекта оценки через страты (блоки, субпотенциалы). На завершающем этапе аналитику лишь необходимо внести ограниченный перечень сведений форм бухгалтерской (финансовой) и статистической отчетности за заданный промежуток времени в рекомендуемую параметрическую кросс-секционную модель оценки экономического потенциала предприятия через субпотенциалы и сравнить результаты расчетов с эталонными (нормативными) уровнями для принятия объективных и своевременных управленческих решений с целью сохранения достигнутых позиций на рынке товаров (работ, услуг). Рекомендуемая универсальная модель расчета интегрального значения экономического потенциала через субпотенциалы син- тезирует традиционный статистический аппарат, метод экспертных оценок и кумулятивную схему компромисса в виде аддитивной свертки, а также ресурсные подходы (потенциалы) к оценке и управлению экономическим потенциалом. Результаты исследования могут быть использованы для повышения предсказуемости рисков и неопределенности ведения бизнеса и придания процессу оценки потенциала системности.

Использование недостаточно обоснованной методики может привести к получению субъективных результатов, которые, в свою очередь, могут стать причиной принятия ошибочных планово-управленческих решений. Роль экономического потенциала в деятельности предприятия настолько велика, что без качественной и количественной оценки потенциала управлять системой невозможно. Оценка экономического потенциала позволяет: конкретизировать имеющиеся возможности предприятия; оценить степень использования экономического потенциала путем сопоставления его величины с эталонным уровнем; определить основные направления проведения корректирующих мероприятий для сохранения и укрепления достигнутых позиций на рынке; определить уровень использования каждого его отдельного элемента для выявления резервных и недоиспользованных возможностей; выявить направления развития экономического потенциала предприятия.

По результатам прохождения этапов аналитик достигает сбалансированности системы оценочных критериев, которая позволяет выявлять и идентифицировать те субпотенциалы, которые не отвечают принципам устойчивости системы к воздействию внешних факторов. В связи с тем, что интегральный показатель (форм.1) 
отражает влияние динамики всех ресурсов конкретного предприятия, можно с полной уверенностью констатировать, что интегральная оценка потенциала отвечает требованиям комплексности, системности, обеспечивает интерпретацию полученных результатов и форми- рование по ним содержательных выводов. Модель позволяет оценить экономический потенциал в динамике, выявить тренды и слабые позиции предприятия в условиях риска и неопределенности на рынке товаров (работ, услуг).

\section{ЛИТЕРАТУРА}

1. Балашова Е.С., Шарипова С. Р. Возможности перехода предприятий к стратегии устойчивого развития // Актуальные проблемы экономики и менеджмента. 2017. № 1. С. 11-16

2. Большой экономический словарь / под.ред. А. Н. Азрилияна.- 2-е изд. доп. и перераб. Москва.: Институт новой экономики, 1997. 864 с.

3. Ожегов С. И. Словарь русского языка: Около 53000 слов / С. И. Ожегов - 25-е изд. Москва.: АЗЬ, 2001. 763 с.

4. Российский энциклопедический словарь: В 2 кн. Кн. 2: Н-Я. / под ред. А. М. Прохорова: Москва.: Большая российская энциклопедия, 2000. 1023 с.

5. Миско К. М. Ресурсный потенциал региона (теоретические и методологические аспекты исследования). Москва.: Наука, 1991. 94 с.

6. Люкшинов А. Н. Стратегический менеджмент на предприятиях АПК. Москва.: Колос, 1999.367 с.

7. Большой коммерческий словарь / под ред. Т. Ф. Рябовой. Москва.: Война и мир, 1996.399 с.

8. Храмцова Т. Г. Методология исследования социально-экономического потенциала потребительской кооперации: дис... д-ра экон. наук.: СибУПК. Новосибирск, 2002.374 с.

9. Клепиков Ю. Н. Оценка уровня и стратегия улучшения использования экономического потенциала предприятия: автореф. дис... канд. экон. наук / Ю. Н. Клепиков Белгород, 1999. 23 с

10. Свободин В. А. Вопросы определения и эффективности производственного потенциала // АПК: экономика, управление. 1991. № 3. С. 27-30.

11. Окорокова Л. Г. Ресурсный потенциал предприятий. СПб.: С-ПбГтУ, 2001. 293 с.

12. Фонотов А. Г. Ресурсный потенциал: планирование, управление. Москва.: Экономика, 1985. 151 с.

13. Смирнова И.В., Смирнова Е.С. Математические модели как основа применения цифровых технологий на предприятиях // Актуальные проблемы экономики и менеджмента. 2019. № 4. С. 135-144

14. Васильев В.Д., Васильев Е. В. Модели расчета «нормы» в рейтинговых схемах. [Электронный ресурс]: Электронный научный журнал Управление экономическими системами. 2013. № 6. Режим доступа: http://uecs.ru/marketing/item/2218—Ir-(дата обращения 17.01.20).

15. Федеральная служба государственной статистики по Тюменской области, Ханты-Мансийскому автономному округу — Югре и Ямало-Ненецкому автономному округу. [Электронный ресурс]. Режим доступа: https://tumstat.gks.ru/. (дата обращения 17.01.20)

16. Сайт Департамента экономики и территориального развития Тюменской области. Электронный ресурс]. Режим доступа: https://economy.admtyumen.ru/. (дата обращения 17.01.20)

17. Метельская Т.В.Оценка инвестиционного риска в анализе инвестиционной привлекательности. [Электронный ресурс]. Режим доступа: elibrary_34896715_45348184. (дата обращения 17.01.20)

18. Боготова 0.Х., Хочуева 3. М., Шеожева Р. Х. Региональная инвестиционная привлекательность и инвестиционный потенциал // Инвестиционная деятельность. Современные тенденции в экономике и управлении. 2014. № 8. С. 105-109. 